Helsinki University of Technology Institute of Mathematics Research Reports

Teknillisen korkeakoulun matematiikan laitoksen tutkimusraporttisarja

Espoo 2004

A468

\title{
ON A TAUBERIAN CONDITION \\ FOR BOUNDED LINEAR OPERATORS
}

J. Malinen, 0. Nevanlinna, and Z. Yuan

TEKNILLINEN KORKEAKOULU

TEKNISKA HÖGSKOLAN

HELSINKI UNIVERSITY OF TECHNOLOGY

TECHNISCHE UNIVERSITÄT HELSINKI

UNIVERSITE DE TECHNOLOGIE D'HELSINKI 

Helsinki University of Technology Institute of Mathematics Research Reports

Teknillisen korkeakoulun matematiikan laitoksen tutkimusraporttisarja

Espoo 2004

A468

\section{ON A TAUBERIAN CONDITION FOR BOUNDED LINEAR OPERATORS}

J. Malinen, 0. Nevanlinna, and Z. Yuan 
J. Malinen, O. Nevanlinna, and Z. Yuan: On a tauberian condition for bounded linear operators; Helsinki University of Technology Institute of Mathematics Research Reports A468 (2004).

Abstract: We study the relation between the growth of sequences $\left\|T^{n}\right\|$ and $\left\|(n+1)(I-T) T^{n}\right\|$ for operators $T \in \mathcal{L}(X)$ satisfying weak variants of the Ritt resolvent condition $\left\|(\lambda-T)^{-1}\right\| \leq \frac{C}{|\lambda-1|}$ for various sets of $|\lambda|>1$.

AMS subject classifications: 47A10, 40E05, 47A30, 47D03

Keywords: Power bounded, Ritt resolvent condition, tauberian theorem

Jarmo.Malinen@hut.fi

ISBN 951-22-7016-1

ISSN 0784-3143

2004

Helsinki University of Technology

Department of Engineering Physics and Mathematics

Institute of Mathematics

P.O. Box 1100, 02015 HUT, Finland

email:math@hut.fi http://www.math.hut.fi/ 


\section{Introduction}

Let $T \in \mathcal{L}(X)$; a bounded linear operator on a (complex) Banach space $X$. It was $\mathrm{R}$. K. Ritt who first studied the Ritt resolvent condition

$$
\left\|(\lambda-T)^{-1}\right\| \leq \frac{C}{|\lambda-1|}
$$

for $|\lambda|>1$. R. K. Ritt himself proved that if $T$ satisfies (1) for $|\lambda|>1$, then $\lim _{n \rightarrow \infty}\left\|T^{n} / n\right\|=0$, see [13]. Clearly (1) implies that $\sigma(T) \subset \mathbb{D} \cup 1$, but in fact even $\sigma(T) \subset K_{\delta}^{c} \cap(\mathbb{D} \cup\{1\})$ for some $\delta>0$, where

$$
K_{\delta}:=\left\{\lambda=1+r e^{i \theta}: r>0 \text { and }|\theta|<\frac{\pi}{2}+\delta\right\}
$$

see O. Nevanlinna [10, Theorem 4.5.4] and Yu. Lyubich [6].

The following result was given by Y. Katznelson and L. Tzafriri in 1986: for power bounded operators $T$ in the sense that $\sup _{n \geq 1}\left\|T^{n}\right\|<\infty$, we have $\sigma(T) \subset \mathbb{D} \cup\{1\}$ if and only if $\lim _{n \rightarrow \infty}\left\|(I-T) T^{n}\right\|=0$, see [5]. Related to this, J. Zemánek asked in 1992 whether (1) implies $\lim _{n \rightarrow \infty}\left\|(I-T) T^{n}\right\|=0$, too. This was answered in positive by O. Nevanlinna, and he also noted that if (1) hold in the larger set $K_{\delta} \cup \mathbb{D}^{c}$ for some $\delta>0$, then $T$ is power bounded, see [10, Theorem 4.5.4], [11] and [16].

It was then observed independently in 1998 by B. Nagy and J. Zemánek [9], O. Nevanlinna, and Yu. Lyubich [6] that if (1) holds for all $|\lambda|>1$, then (1), indeed, holds for all $\lambda \in K_{\delta} \cup \mathbb{D}^{c}$ for some $\delta>0$ (with another possibly larger constant $\tilde{C}$ in place for $C$ ). Hence, if $T$ satisfies (1) for all $|\lambda|>1$, then $T$ is power bounded. The upper bound $\sup _{n \geq 1}\left\|T^{n}\right\| \leq\left(e C^{2}\right) / 2$ was given by N. Borovykh, D. Drissi and M. N. Spijker, see [1]. A tighter estimate $\sup _{n \geq 1}\left\|T^{n}\right\| \leq C^{2}$ was shown by O. El-Fallah and T. Ransford in [2].

Much of these developments culminate in the following fundamental result connecting power boundedness, the Ritt resolvent condition and the tauberian condition (3):

Proposition 1. The following are equivalent:

(i) T satisfies (1) for all $|\lambda|>1$,

(ii) $\sigma(T) \subset \mathbb{D} \cup\{1\}$ and $T$ satisfies (1) for all $\lambda \in K_{\delta}$ for some $\delta>0$, and

(iii) $T$ is power bounded, and it satisfies the tauberian condition

$$
\sup _{n \geq 1}(n+1)\left\|(I-T) T^{n}\right\| \leq M
$$

for some $M<\infty$.

Indeed, the equivalence (i) $\Leftrightarrow$ (ii) has already been discussed above. That (ii) $\Rightarrow$ (iii) is given in [10, Theorem 4.5.4], and we shall compute an estimate for $M$ in (3) in Theorem 4 . That (iii) implies (i) was reported in [11, Theorem 
2.1]. The proof relies on the theory of analytic semigroups, and it follows closely $[12 \text {, Theorem } 5.2]^{1}$.

We further note that J. Esterle has pointed out in [3] that

$$
\liminf _{n \rightarrow \infty}(n+1)\left\|(I-T) T^{n}\right\| \geq \frac{1}{96}
$$

for a power-bounded $T$ satisfying $\sigma(T)=\{1\}$; see also [8] (and references therein) for the determination of the optimal lower bound $1 / e$ instead of $1 / 96$. Hence the stronger version $\lim _{n \rightarrow \infty}(n+1)(I-T) T^{n}=0$ of the tauberian condition (3) cannot generally hold for $T$ satisfying (1) for all $|\lambda|>1$.

We shall show in this paper that the conditions of Proposition 1 can be combined in a different way. Indeed, we shall prove the following tauberian theorem and discuss some of its consequences:

Theorem 1. If $T \in \mathcal{L}(X)$ satisfies the the Ritt condition (1) for all $\lambda>1$ and tauberian condition (3), then $T$ is power bounded.

We also estimate $\sup _{n \geq 1}\left\|(n+1)(I-T) T^{n}\right\|$ for operators satisfying (1) for all $|\lambda|>1$. Most of the results of this paper (in particular, the main result Theorem 2) were proved in 2002 in [15].

\section{Equivalent conditions under the tauberian condition}

Let us remind the results of the classical tauberian theorem in the scalar case. Let $\left\{a_{n}\right\}$ be a complex sequence and $s_{n}=a_{0}+a_{1}+\ldots+a_{n}$ for $n \geq 0$. A. Tauber proved in 1897 that if

(i) $\lim _{n \rightarrow \infty}(n+1) a_{n}=0$, and

(ii) $\lim _{r \rightarrow 1_{-}} f(r)=s$, where $f(r)=\sum_{0}^{\infty} a_{n} r^{n}$ for $0<r<1$,

then $\lim _{n \rightarrow \infty} s_{n}=s$. It was J. E. Littlewood who later in 1910 showed that the tauberian condition (i) can in fact be replaced by the weaker tauberian condition $\sup _{n} n\left|a_{n}\right|<\infty$. As it is mentioned in [14, Chapter 9], the proof with this modification becomes considerable harder.

If we take $a_{n}=(I-T) T^{n}$, we see that the weaker tauberian condition is exactly (3). Now the corresponding partial sums are simply $s_{n}=I-T^{n+1}$. In this paper, we are not interested in the limit behaviour of $\left\{s_{n}\right\}$, but only in the boundedness of this sequence under the weaker tauberian condition (3). This will save us from the extra complications that would be required if we had to take advantage of Littlewood's variant of the classical tauberian theorem instead.

\footnotetext{
${ }^{1}$ However, the restrictive assumption $0 \in \rho(A)$ must be first removed from [12, Theorem $5.2]$ by a more careful analysis.
} 
Theorem 2. Assume that $T \in \mathcal{L}(X)$ satisfies tauberian condition (3), and

$$
\left\|(\lambda-1)(\lambda-T)^{-1}\right\| \leq C
$$

for all $\lambda>1$. Then $T$ is power bounded with the estimates

$$
\begin{aligned}
& \left\|T^{n}\right\| \leq 2+C\|T\|+2 M \quad \text { and } \\
& \limsup _{n \rightarrow \infty}\left\|T^{n}\right\| \leq 2+C\|T\|+(1+1 / e) M .
\end{aligned}
$$

Proof. Define

$$
\begin{aligned}
s_{n} & :=\sum_{j=0}^{n-1}(I-T) T^{j}=1-T^{n}, \\
f(r) & :=\sum_{j=0}^{\infty}(I-T) T^{j} r^{j}=(I-T)(1-r T)^{-1}, \text { and } \\
f_{n}(r) & :=\sum_{j=0}^{n-1}(I-T) T^{j} r^{j} .
\end{aligned}
$$

Then for all $r \in(0,1)$ and $n \geq 0$, we have

$$
\left\|s_{n}\right\| \leq\left\|s_{n}-f_{n}(r)\right\|+\left\|f_{n}(r)-f(r)\right\|+\|f(r)\| .
$$

Condition (4) implies $\sup _{0 \leq r<1}\|f(r)\| \leq 1+C\|T\|$, and the last term of the right hand side is bounded by $C_{1}:=1+C\|T\|$. For the second term, we have

$$
\begin{aligned}
& \left\|f_{n}(r)-f(r)\right\|=\left\|\sum_{j \geq n}(I-T) T^{j} r^{j}\right\| \leq \sum_{j \geq n} \frac{M}{j+1} r^{j} \\
& =\frac{M}{n+1} \sum_{j \geq n} \frac{n+1}{j+1} r^{j} \leq \frac{M}{n+1} r^{n}(1-r)^{-1}
\end{aligned}
$$

by (3). From now on, we choose $r_{n}:=1-1 / n$ in (5). Then

$$
\frac{M}{n+1} r_{n}^{n}\left(1-r_{n}\right)^{-1}=\frac{M}{n+1}\left(1-\frac{1}{n}\right)^{n} n \quad\left\{\begin{array}{l}
\rightarrow M / e \text { as } n \rightarrow \infty \\
\leq M \text { for all } n \geq 1
\end{array}\right.
$$

So the second term in (5) is bounded with this choice of $r=r_{n}$.

The first term of the right side of inequality (5) (when choosing $r=r_{n}$ ) we have

$$
s_{n}-f_{n}\left(r_{n}\right)=\sum_{j=0}^{n-1}(I-T) T^{j}\left(1-r_{n}^{j}\right) .
$$

By the mean value theorem, there exists $r_{0}^{j} \in\left[r_{n}, 1\right)$ for any $j>0$, such that we can estimate

$$
1-r_{n}^{j}=j r_{0}^{j-1}\left(1-r_{n}\right) \leq j\left(1-r_{n}\right)=\frac{j}{n}
$$


This together with (3) yields

$$
\begin{gathered}
\left\|s_{n}-f_{n}\left(r_{n}\right)\right\| \leq \sum_{j=0}^{n-1} \frac{j}{n}\left\|(I-T) T^{j}\right\| \\
\leq \sum_{j=0}^{n-1} \frac{j}{n} \frac{M}{j+1} \leq M \frac{1}{n} \sum_{j=0}^{n-1} 1=M .
\end{gathered}
$$

So $\left\|s_{n}\right\|$ is uniformly bounded, which is equivalent to the power boundedness of $T$. This completes the proof.

If the tauberian condition (3) holds for $T$, then a number of conditions will be equivalent. The following theorem is analogous to [11, Theorem 2.1], except that now (3) is a standing assumption instead of power boundedness.

Theorem 3. Assume that $T \in \mathcal{L}(X)$ satisfies the tauberian condition (3). Then the following are equivalent:

(i) $T$ is power bounded,

(ii) $T$ satisfies Kreiss resolvent condition for some constant $C_{K}$

$$
\left\|(\lambda-T)^{-1}\right\| \leq \frac{C_{K}}{|\lambda|-1}
$$

for $|\lambda|>1$,

(iii) there exists $0<\eta \leq 1 \leq C<\infty$ such that $T$ satisfies the Ritt resolvent condition (1) for all real $\lambda \in(1,1+\eta)$,

(iv) there exists $0<\eta \leq 1 \leq C<\infty$ such that $T$ satisfies the second order Ritt condition

$$
\left\|(\lambda-1)^{2}(\lambda-T)^{-2} T\right\| \leq C,
$$

for all real $\lambda \in(1,1+\eta)$,

(v) there exists $0<\delta \leq 1 \leq C<\infty$ such that $T$ satisfies the Ritt resolvent condition (1) for all $\lambda \in K_{\delta}^{\prime}:=\left\{\lambda=1+r e^{i \theta}|r>0,| \theta \mid<\frac{\pi}{2}+\delta\right\}$, and

(vi) $A:=T-I$ generates an uniformly bounded, norm continuous, analytic semigroup $t \mapsto e^{A t}$ of linear operators.

Proof. It is shown by estimating the von Neumann series that (i) $\Rightarrow$ (ii). It is trivial that (ii) $\Rightarrow$ (iii), and (iii) $\Rightarrow$ (i) by Theorem 2 , noting that the resolvent condition is only used near point 1 in the proof.

It is trivial that (iii) implies (iv). Conversely, noting that because $\sigma(T) \subset$ $\overline{\mathbb{D}}$ by the tauberian condition (3), we obtain for all $|r|<1$

$$
(I-r T)^{-1}=\sum_{j \geq 0}(j+1)(I-T) T^{j} r^{j}+(1-r)(I-r T)^{-2} T .
$$


From this we conclude, by using (3) in the estimation, that

$$
\begin{aligned}
& \left\|(1-r)(I-r T)^{-1}\right\| \leq(1-r) \cdot M \sum_{j \geq 0} r^{j}+\left\|(1-r)^{2}(I-r T)^{-2} T\right\| \\
& =M+\left\|(1-r)^{2}(I-r T)^{-2} T\right\|
\end{aligned}
$$

for all $0 \leq r<1$. Replacing $r=1 / \lambda$ shows now that (iv) $\Rightarrow$ (iii).

Claims (i) and (v) are equivalent by Proposition 1 and the extension result that can be found e.g. in [9]. By the classical theorem of E. Hille and K. Yoshida, claim (v) is equivalent (apart from the analyticity of the semigroup) to the existence of $C_{H Y}<\infty$ such that for each integer $k \geq 1$

$$
\left\|(\lambda-T)^{-k}\right\| \leq \frac{C_{H Y}}{(\lambda-1)^{k}} \quad \text { for all } \quad \lambda>1 .
$$

Setting $k=1$ gives (iii). Conversely, (i) $\Rightarrow$ (vi) (apart from the analyticity) by the estimate

$$
\left\|e^{t T}\right\| \leq \sum_{j \geq 0} \frac{\left\|T^{j}\right\| t^{j}}{j !} \leq \sup _{j \geq 0}\left\|T^{j}\right\| \cdot e^{t}
$$

for all $t \geq 0$. Moreover, it is not difficult to see that $\left\|A e^{t A}\right\| \leq M t^{-1}\left(1-e^{-t}\right)$ where $A:=T-I$, if (3) holds. This implies that $e^{t A}$ is analytic, by a slight generalization of $[12$, Theorem 5.2].

The implication (i) $\Rightarrow$ (ii) (with explicit constants) was first given by Z. Yuan by using a Cauchy integration argument, see [15]. We remark that the tauberian condition (3) implies $\left\|T^{n}\right\|=O(\ln n)$, and by $[4$, Theorem 3.3], [7], the growth can really be there for an operator in a Banach space. Condition (3) "almost" implies condition (iii) of Theorem 3, too. Indeed, as $(1-r)(I-r T)^{-1}=I-r(I-T)(I-r T)^{-1}$ for all $|r|<1$, we obtain the estimate

$$
\left\|(1-r)(I-r T)^{-1}\right\| \leq 1+M \sum_{j \geq 0} \frac{|r|^{j+1}}{j+1}=1+M \ln \frac{1}{1-|r|}
$$

for all $0 \leq|r|<1$. Setting $r=1 / \lambda$ for $\lambda>1$ gives now

$$
\left\|(\lambda-1)(\lambda-T)^{-1}\right\| \leq 1+M \ln \frac{\lambda}{\lambda-1} .
$$

Hence $\left\|(\lambda-T)^{-1}\right\|=O((\lambda-1) \ln (\lambda-1))$ as $\lambda \rightarrow 1+$. Again, the logarithmic term can really be present on the right hand side, see [7].

Finally, the tauberian condition (3) "almost" implies condition (vi) of Theorem 3. Indeed, as $\left\|A e^{t A}\right\| \leq M t^{-1}\left(1-e^{-t}\right)$ where $A:=T-I$, and the function $t \mapsto t^{-1}\left(1-e^{-t}\right)$ is decreasing for $t \geq 0$, it follows that

$$
\left\|e^{t A}\right\| \leq 1+\int_{0}^{t}\left\|A e^{t A}\right\| d t \leq 1+M+M\left(1-e^{-1}\right) \ln t \quad \text { for all } \quad t \geq 1 .
$$




\section{An upper bound for $\left\|(n+1)(I-T) T^{n}\right\|$}

Assume that $T \in \mathcal{L}(X)$ satisfies the Ritt resolvent condition (1) for all $|\lambda|>$ 1. Then $\sup _{n \geq 1}\left\|T^{n}\right\| \leq C^{2}$, as shown in [2] as a particular case of a much more general result. The earlier upper bound $\sup _{n \geq 1}\left\|T^{n}\right\| \leq\left(e C^{2}\right) / 2$ was given in [1]. We proceed to give a common upper bound for the operators $n(I-T) T^{n}$ appearing in the tauberian condition (3).

Theorem 4. Assume that $T \in \mathcal{L}(X)$ satisfies (1) for all $|\lambda|>1$. Then

$$
\sup _{n \geq 1}(n+1)\left\|(I-T) T^{n}\right\| \leq 2 \sup _{n \geq 2}\left\|T^{n}\right\|+e C^{3} .
$$

Proof. Recall that we have by the Cauchy interal

$$
(I-T) T^{n}=\frac{1}{2 \pi i} \int_{\Gamma} \lambda^{n}(1-\lambda)(\lambda-T)^{-1} d \lambda,
$$

where $\Gamma$ is an arbitrary positively oriented circle $|\lambda|=r>1$. By partially integrating twice, we obtain

$$
\begin{aligned}
& (I-T) T^{n}=\frac{1}{\pi i(n+1)(n+2)} \int_{\Gamma} \lambda^{n+2}(1-\lambda)(\lambda-T)^{-3} d \lambda \\
& +\frac{1}{n+1} \cdot \frac{2}{\pi i(n+2)(n+3)} \int_{\Gamma} \lambda^{n+3}(\lambda-T)^{-3} d \lambda .
\end{aligned}
$$

By partially integrating twice the Cauchy integral representation, we get

$$
T^{n+1}=\frac{1}{\pi i(n+2)(n+3)} \int_{\Gamma} \lambda^{n+3}(\lambda-T)^{-3} d \lambda .
$$

So we have for all $n \geq 1$

$$
(n+1)(I-T) T^{n}-2 T^{n+1}=\frac{1}{\pi i(n+2)} \int_{\Gamma} \lambda^{n+2}(1-\lambda)(\lambda-T)^{-3} d \lambda .
$$

By the Ritt resolvent condition (1) we get $\left\|(1-\lambda)(\lambda-T)^{-3}\right\| \leq C^{3}|1-\lambda|^{-2}$ and hence for all $r>1$

$$
\left\|(n+1)(I-T) T^{n}-2 T^{n+1}\right\| \leq \frac{r^{n+2} C^{3} J}{(n+2) \pi}
$$

where after computations

$$
J=\int_{-\pi}^{\pi} \frac{r d t}{\left|r e^{i t}-1\right|^{2}}=\frac{2 \pi r}{r^{2}-1} .
$$

Inserting the above expression for $J$ into (8), we get

$$
\left\|(n+1)(I-T) T^{n}-2 T^{n+1}\right\| \leq 2 C^{3} F(n, r),
$$


where $F(n, r):=\frac{r^{n+3}}{(n+2)\left(r^{2}-1\right)}$ for all $r>1$ and $n \geq 1$. Moreover,

$$
\min _{r>1} F(n, r)=F\left(n, \sqrt{1+\frac{2}{n+1}}\right)=\frac{n+3}{2(n+2)}\left(1+\frac{2}{n+1}\right)^{\frac{n+1}{2}}
$$

and after rather long computations that we omit here, we get finally $\sup _{n \geq 1} F(n, \sqrt{1+2 /(n+1)})=e / 2$. These together with (9) prove the claim.

By letting $|\lambda| \rightarrow \infty$, it is easy to see that necessarily $C \geq 1$ in (1). Using this together with the bounds $\sup _{n \geq 1}\left\|T^{n}\right\| \leq C^{2}$ and (7) gives a more simple upper bound $\sup _{(n+1) \geq 1}(n+1)\left\|(\bar{I}-T) T^{n}\right\| \leq(2+e) C^{3}$. In fact, the proof of Theorem 4 shows that the boundedness of sequences $\left\{T^{n}\right\}$ and $\left\{(n+1)(I-T) T^{n}\right\}$ is equivalent, whenever $T$ satisfies only the "third order" Ritt condition

$$
\sup _{|\lambda|>1}\left\|(1-\lambda)^{3}(\lambda-T)^{-3}\right\|<\infty
$$

\section{References}

[1] N. Borovykh, D. Drissi, and M. N. Spijker. A note about Ritt's condition and related resolvent conditions. Numerical Functional Analysis and Optimization, 21(3-4):425-438, 2000.

[2] O. El-Fallah and T. Ransford. Extremal growth of powers of operators satisfying resolvent conditions of Kreiss-Ritt type. Journal of Functional Analysis, 196:135-154, 2002.

[3] J. Esterle. Quasimultipliers, representations of $H^{\infty}$, and the closed ideal problem for commutative Banach algebras. In Radical Banach algebras and automatic continuity (Long Beach, Calif., 1981), volume 975 of Lecture Notes in Mathematics, pages 66-162, Berlin, 1983. Springer Verlag.

[4] N. Kalton, S. Montgomery-Smith, K. Oleszkiewicz, and Y. Tomilov. Power-bounded operators and related norm estimates. Preprint, 2002.

[5] Y. Katznelson and L. Tzafriri. On power bounded operators. Journal of Functional Analysis, 68:313-328, 1986.

[6] Yu. Lyubich. Spectral localization, power boundedness and invariant subspaces under Ritt's type condition. Studia Mathematica, 143(2):153167, 1999.

[7] O. E. Maasalo, J. Malinen, and V. Turunen. An example of an operator satisfying a tauberian condition. Preprint, Helsinki University of Technology. 
[8] J. Malinen, O. Nevanlinna, V. Turunen, and Z. Yuan. A lower bound for the differences of powers of linear operators. Helsinki University of Technology Institute of Mathematics Research Reports, A467, 2004.

[9] B. Nagy and J. Zemánek. A resolvent condition implying power boundedness. Studia mathematica, 132(2):143-151, 1999.

[10] O. Nevanlinna. Convergence of Iterations for Linear Equations. Lectures in Mathematics ETH Zürich. Birkhäuser Verlag, Basel, Boston, Berlin, 1993.

[11] O. Nevanlinna. On the growth of the resolvent operators for power bounded operators. In F.H. Szafraniec J. Janas and J. Zemánek, editors, Linear Operators, volume 38 of Banach Center Publications, pages 247264. Inst. Math., Polish Acad. Sci., 1997.

[12] A. Pazy. Semigroups of linear operators and applications to partial differential equations, volume 44 of Applied Mathematical Sciences. Springer Verlag, 1983.

[13] R. K. Ritt. A condition that $\lim _{n \rightarrow \infty} n^{-1} T^{n}=0$. Proc. Amer. Math. Soc., 4:898-899, 1953.

[14] W. Rudin. Functional Analysis. McGraw-Hill Book Company, New York, TMH edition, 1990.

[15] Z. Yuan. On the resolvent and Tauberian conditions for bounded linear operators. Licentiate's Thesis. Helsinki University Of Technology, March 2002.

[16] J. Zemanek. On the Gelfand-Hille theorems. In J. Zemanek, editor, Functional Analysis and Operator Theory, volume 30, pages 369-385. Banach center publications, 1994. 
(continued from the back cover)

A460 Timo Eirola, Jan von Pfaler

Numerical Taylor expansions for invariant manifolds

April 2003

A459 Timo Salin

The quenching problem for the $\mathrm{N}$-dimensional ball

April 2003

A458 Tuomas Hytönen

Translation-invariant Operators on Spaces of Vector-valued Functions

April 2003

A457 Timo Salin

On a Refined Asymptotic Analysis for the Quenching Problem

March 2003

A456 Ville Havu, Harri Hakula, Tomi Tuominen

A benchmark study of elliptic and hyperbolic shells of revolution

January 2003

A455 Yaroslav V. Kurylev, Matti Lassas, Erkki Somersalo

Maxwell's Equations with Scalar Impedance: Direct and Inverse Problems

January 2003

A454 Timo Eirola, Marko Huhtanen, Jan von Pfaler

Solution methods for R-linear problems in $C^{n}$

October 2002

A453 Marko Huhtanen

Aspects of nonnormality for iterative methods

September 2002

A452 Kalle Mikkola

Infinite-Dimensional Linear Systems, Optimal Control and Algebraic Riccati

Equations

October 2002 


\section{HELSINKI UNIVERSITY OF TECHNOLOGY INSTITUTE OF MATHEMATICS RESEARCH REPORTS}

The list of reports is continued inside. Electronical versions of the reports are available at $h$ ttp://www.math.hut.fi/reports/ .

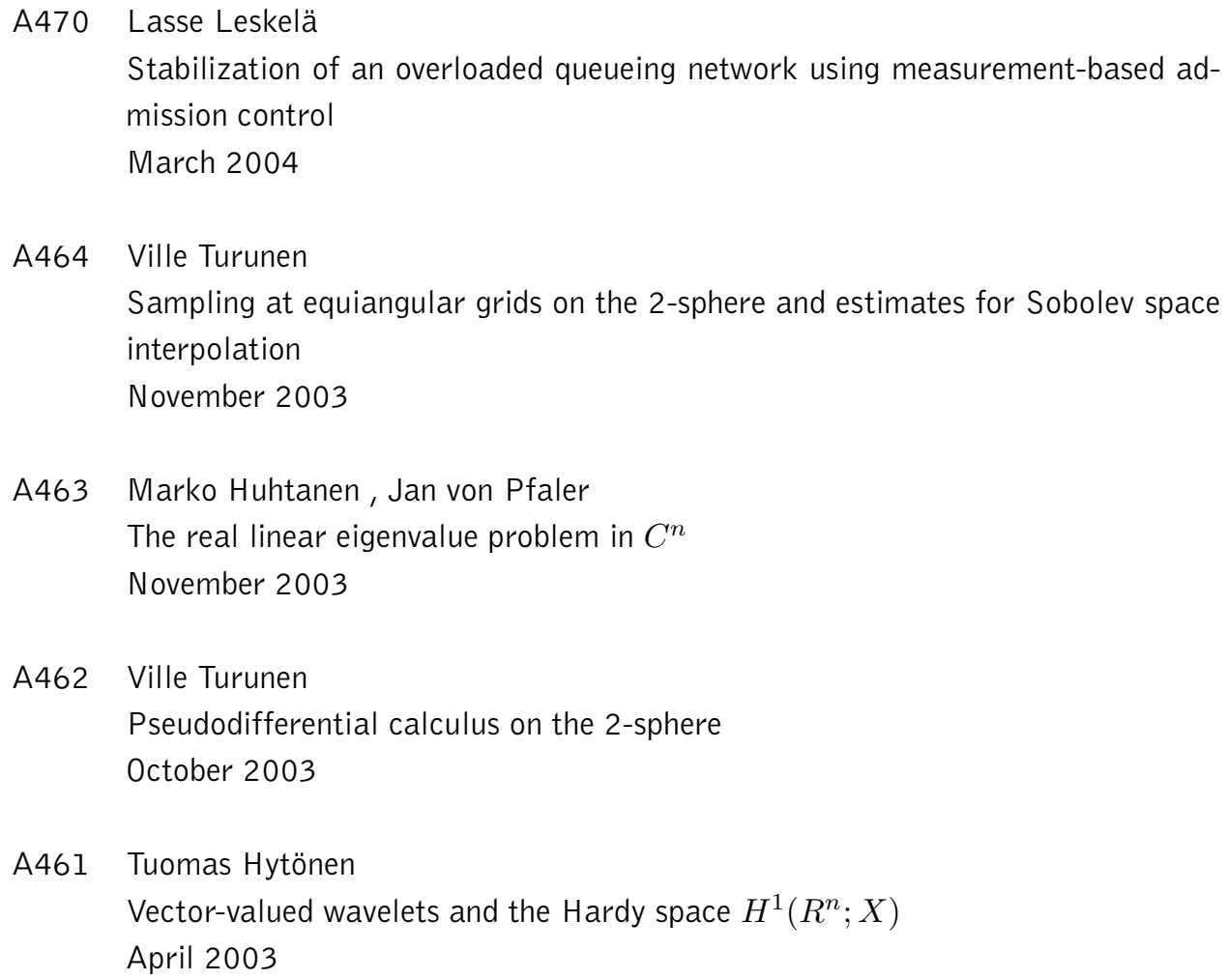

